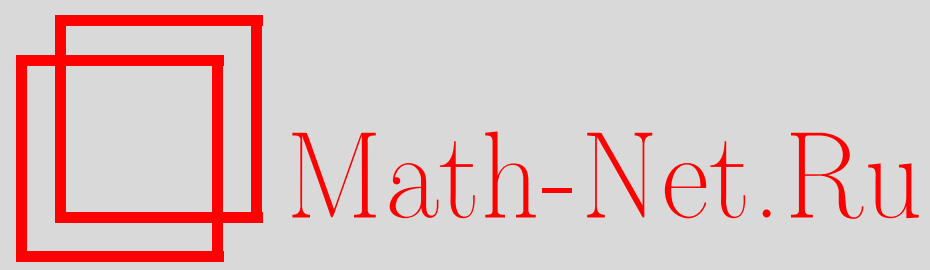

И. И. Шарапудинов, Об одном новом применении многочленов Чебышева, ортогональных на равномерной сетке, $M a$ тем. заметки, 1998, том 64, выпуск 6, 950-953

DOI: https://doi.org/10.4213/mzm1475

Использование Общероссийского математического портала Math-Net.Ru подразумевает, что вы прочитали и согласны с пользовательским соглашением http://www . mathnet.ru/rus/agreement

Параметры загрузки:

IP: 54.205 .225 .156

26 апреля 2023 г., 13:04:41

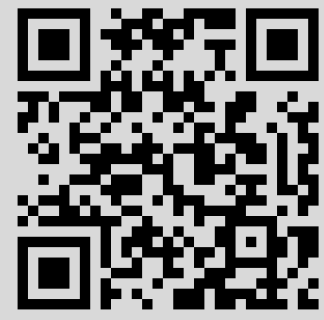




\section{ОБ ОДНОМ НОВОМ ПРИМЕНЕНИИ МНОГОЧЛЕНОВ ЧЕБЫШЕВА, ОРТОГОНАЛЬНЫХ НА РАВНОМЕРНОЙ СЕТКЕ}

\section{И.И. Шарапудинов}

1. В связи с некоторыми вопросами теории преобразований Радона К. И. Осколковым была поставлена задача об исследовании поведения величины

$$
q(n, N)=\inf _{p_{n} \in \widehat{H}_{n}} \sum_{j=1}^{N} p_{n}^{2}(j),
$$

где $\widehat{H}_{n}$ - множество алгебраических многочленов степени $n$, удовлетворяющих условию $p_{n}(0)=1$. В частности, требуется найти условие на рост степени $n$ многочлена $p_{n}(x)\left(p_{n}(0)=1\right)$, которое обеспечивает стремление величины $q(n, N)$ к 0 при $N \rightarrow \infty$. Оказалось, что это условие состоит в том, что $n / \sqrt{N} \rightarrow \infty$. Причем оно окончательно в том смысле, что если $n=O(\sqrt{N})$, то $q(n, N) \geqslant c>0$. Имеет место

TеОРема. Пусть а - фиксированное положительное число. Тогда если $n \leqslant a \sqrt{N}$, то найдется постоянная $c=c(a)>0$, для которой

$$
q(n, N) \geqslant c .
$$

Ecли же $n / \sqrt{N} \rightarrow \infty$, mo

$$
\lim _{N \rightarrow \infty} q(n, N)=0
$$

более точно,

$$
\begin{array}{ll}
q(n, N) \leqslant \frac{2 e^{1 / 24} N}{n(n-1)+2 n \exp \left\{3 n^{2} / 20 N\right\}}, & n \leqslant \frac{N}{4} \\
q(n, N) \leqslant \frac{32 e^{1 / 24}}{N-4+8 \exp \{3 N / 320\}}, & n>\frac{N}{4} .
\end{array}
$$

2. При доказательстве теоремы нам понадобятся некоторые сведения о многочленах Чебьшева $T_{n}^{\alpha, \beta}(x, N)(0 \leqslant n \leqslant N-1)$, ортогональных на сетке $\Omega=\{0,1, \ldots, N-1\}$. Для $\alpha, \beta>-1$ положим

$$
\begin{gathered}
\mu(x)=\frac{\Gamma(N) 2^{\alpha+\beta+1}}{\Gamma(N+\alpha+\beta+1)} \frac{\Gamma(x+\beta+1) \Gamma(N-x+\alpha)}{\Gamma(x+1) \Gamma(N-x)}, \\
h_{n, N}^{\alpha, \beta}=\frac{(N+n+\alpha+\beta)^{[n]}}{(N-1)^{[n]}} \frac{\Gamma(n+\alpha+1) \Gamma(n+\beta+1) 2^{\alpha+\beta+1}}{n ! \Gamma(n+\alpha+\beta+1)(2 n+\alpha+\beta+1)},
\end{gathered}
$$

где $a^{[0]}=1, a^{[n]}=a(a-1) \cdots(a-n+1)(n \geqslant 1)$. Тогда [1]

$$
\sum_{x=0}^{N-1} \mu(x) T_{n}^{\alpha, \beta}(x, N) T_{m}^{\alpha, \beta}(x, N)=\delta_{n m} h_{n, N}^{\alpha, \beta}
$$

Работа выполнена при финансовой поддержке Российского фонда фундаментальных исследований, грант № 98-01-00930. 
и имеет место формула

$$
T_{n}^{\alpha, \beta}(x, N)=(-1)^{n} \frac{\Gamma(n+\beta+1)}{n !} \sum_{k=0}^{n}(-1)^{k} \frac{n^{[k]}(n+\alpha+\beta+1)_{k} x^{[k]}}{\Gamma(k+\beta+1) k !(N-1)^{[k]}},
$$

где $(a)_{0}=1,(a)_{k}=a(a+1) \cdots(a+k-1)(k \geqslant 1)$.

Из (8) следует, что многочлены

$$
\tau_{n}^{\alpha, \beta}(x)=\tau_{n}^{\alpha, \beta}(x, N)=\left(h_{n, N}^{\alpha, \beta}\right)^{-1 / 2} T_{n}^{\alpha, \beta}(x, N), \quad 0 \leqslant n \leqslant N-1,
$$

образуют на сетке $\Omega=\{0,1, \ldots, N-1\}$ ортонормированную систему с весом $\mu(x)$, т.е.

$$
\sum_{x=0}^{N-1} \mu(x) \tau_{n}^{\alpha, \beta}(x, N) \tau_{m}^{\alpha, \beta}(x, N)=\delta_{n m}, \quad 0 \leqslant n, m \leqslant N-1 .
$$

Пусть $x_{0}$ - произвольное число, $b_{n}(x)$ - произвольньй многочлен степени $n$, нормированный условием

$$
\sum_{x=0}^{N-1} \mu(x)\left(b_{n}(x)\right)^{2}=1
$$

Тогда максимум $\left(b_{n}\left(x_{0}\right)\right)^{2}$ достигается для многочленов

$$
b_{n}(x)=\varepsilon\left(\mathscr{K}_{n, N}^{\alpha, \beta}\left(x_{0}, x_{0}\right)\right)^{-1 / 2} \mathscr{K}_{n, N}^{\alpha, \beta}\left(x_{0}, x\right), \quad|\varepsilon|=1,
$$

где

$$
\mathscr{K}_{n, N}^{\alpha, \beta}\left(x_{0}, x\right)=\tau_{0}^{\alpha, \beta}\left(x_{0}\right) \tau_{0}^{\alpha, \beta}(x)+\tau_{1}^{\alpha, \beta}\left(x_{0}\right) \tau_{1}^{\alpha, \beta}(x)+\cdots+\tau_{n}^{\alpha, \beta}\left(x_{0}\right) \tau_{n}^{\alpha, \beta}(x) .
$$

Этот максимум равен

$$
\left(b_{n}\left(x_{0}\right)\right)^{2}=\mathscr{K}_{n}^{\alpha, \beta}\left(x_{0}, x_{0}\right)=\sum_{k=0}^{n}\left(\tau_{k}^{\alpha, \beta}\left(x_{0}\right)\right)^{2} .
$$

3. Нам понадобятся некоторые оценки для величины $\mathscr{K}_{n}^{\alpha, \beta}\left(x_{0}, x_{0}\right)$ при $\alpha=\beta=0, x_{0}=-1$. Полагая $T_{n}(x, N)=T_{n}^{0,0}(x, N)$, из (9) имеем

$$
T_{n}(-1, N)=(-1)^{n} \sum_{k=0}^{n}(-1)^{k} \frac{n^{[k]}(n+1)_{k}(-1)^{[k]}}{k !^{2}(N-1)^{[k]}}=(-1)^{n} \sum_{k=0}^{n} \frac{n^{[k]}(n+1)_{k}}{k !(N-1)^{[k]}} \text {. }
$$

Отсюда

$$
\begin{aligned}
\left|T_{n}(-1, N)\right| & =\sum_{k=0}^{n}\left(\begin{array}{l}
n \\
k
\end{array}\right) \frac{(n+1)_{k}}{(N-1)^{[k]}}>\sum_{k=0}^{n}\left(\begin{array}{l}
n \\
k
\end{array}\right)\left(\frac{n}{N}\right)^{k}=\left(1+\frac{n}{N}\right)^{n} \\
& >\exp \left\{\frac{n^{2}}{N}-\frac{n^{3}}{2 N^{2}}\right\}, \quad n \leqslant N-1 .
\end{aligned}
$$

С другой стороны, из (7) имеем

$$
\begin{aligned}
\left(h_{n, N}^{\alpha, \beta}\right)^{-1} & =\frac{(N-n) \cdots(N-1)}{N(N+1) \cdots(N+n-1)} \frac{N(2 n+1)}{2(N+n)}=\frac{N(2 n+1)}{2(N+n)} \exp \left\{\sum_{k=0}^{n-1} \ln \left(1-\frac{n}{N+k}\right)\right\} \\
& =\frac{N(2 n+1)}{2(N+n)} \exp \left\{-\sum_{k=0}^{n-1} \sum_{j=1}^{\infty} \frac{n^{j}}{j(N+k)^{j}}\right\} \\
& =\frac{N(2 n+1)}{2(N+n)} \exp \left\{-\sum_{k=0}^{n-1} \frac{n}{N+k}-\sum_{j=2}^{\infty} \frac{n^{j}}{j} \sum_{k=0}^{n-1} \frac{1}{(N+k)^{j}}\right\} .
\end{aligned}
$$


Если $j \geqslant 2$, то

$$
\sum_{k=0}^{n-1} \frac{1}{(N+k)^{j}}<\int_{0}^{n-1} \frac{d x}{(N+x)^{j}}+\frac{1}{N^{j}}<\frac{1}{N^{j}}+\frac{1}{j-1}\left(\frac{1}{N^{j-1}}-\frac{1}{(N+n)^{j-1}}\right) .
$$

Отсюда и из (15) находим

$$
\left(h_{n, N}^{0,0}\right)^{-1}>\frac{N(2 n+1)}{2(N+n)} \exp \left\{-\frac{n^{2}}{N}-\sum_{j=2}^{\infty} \frac{n^{j}}{j(j-1) N^{j-1}}-\sum_{j=2}^{\infty} \frac{n^{j}}{j N^{j}}+\sum_{j=2}^{\infty} \frac{n^{j}}{j(j-1)(N+n)^{j-1}}\right\} .
$$

Далее,

$$
\begin{gathered}
\sum_{j=2}^{\infty} \frac{n^{j}}{j N^{j}}<\frac{1}{2}\left(\frac{n}{N}\right)^{2} \sum_{j=0}^{\infty}\left(\frac{n}{N}\right)^{j}<\frac{1}{2}\left(\frac{n}{N}\right)^{2} \frac{N}{N-n}=\frac{n^{2}}{2 N(N-n)}, \quad n \leqslant N-1, \\
\sum_{j=2}^{\infty} \frac{n^{j}}{j(j-1) N^{j-1}}<\frac{n^{2}}{N} \sum_{j=2}^{\infty} \frac{1}{j(j-1)}=\frac{n^{2}}{N}, \quad n \leqslant N-1, \\
\sum_{j=2}^{\infty} \frac{n^{j}}{j(j-1)(N+n)^{j-1}}>\frac{n^{2}}{2(N+n)}, \quad n \leqslant N-1 .
\end{gathered}
$$

Сопоставляя (16)-(19), получаем

$$
\left(h_{n, N}^{0,0}\right)^{-1 / 2}>\sqrt{\frac{N(2 n+1)}{2(N+n)}} \exp \left\{-\frac{n^{2}}{N}-\frac{n^{2}}{4 N(N-n)}+\frac{n^{2}}{4(N+n)}\right\} .
$$

Из (10), (14) и (20) выводим

$$
\left|\tau_{n}^{0,0}(-1, N)\right|>\sqrt{\frac{N(2 n+1)}{2(N+n)}} \exp \left\{\frac{n^{2}}{4(N+n)}-\frac{n^{3}}{2 N^{2}}-\frac{n^{2}}{4 N(N-n)}\right\}, \quad 0 \leqslant n \leqslant N-1 .
$$

Из (13) и (21) мы находим следующую оценку:

$$
\mathscr{K}_{n, N}^{0,0}(-1,-1)>\sum_{k=0}^{n} \frac{N(2 k+1)}{2(N+k)} \exp \left\{\frac{k^{2}}{2(N+k)}-\frac{k^{3}}{N^{2}}-\frac{k^{2}}{2 N(N-k)}\right\}, \quad 0 \leqslant n \leqslant N-1 .
$$

Нам понадобится также оценка сверху для $\mathscr{K}_{n, N}^{0,0}(-1,-1)$ в случае $n=O(\sqrt{N})$. Из первого соотношения (14) имеем

$$
\begin{aligned}
\left|T_{n}(-1, N)\right| & <\sum_{k=0}^{n}\left(\begin{array}{l}
n \\
k
\end{array}\right)\left(\frac{2 n}{N-n}\right)^{k}=\left(1+\frac{2 n}{N-n}\right)^{n} \\
& =\left(1+\frac{2 n}{N-n}\right)^{(N-n) /(2 n) \cdot 2 n^{2} /(N-n)}<e^{2 n^{2} /(N-n)}
\end{aligned}
$$

а из (15) вьводим

$$
\left(h_{n, N}^{0,0}\right)^{-1} \leqslant \frac{2 n+1}{2} .
$$

Стало быть,

$$
\left|\tau_{n}^{0,0}(-1, N)\right|=\left(h_{n, N}^{0,0}\right)^{-1 / 2}\left|T_{n}^{0,0}(-1, N)\right| \leqslant \sqrt{\frac{2 n+1}{2}} e^{2 n^{2} /(N-n)} .
$$

Если число $a>0$ фиксировано и $n \leqslant a \sqrt{N}$, то из (23) находим

$$
\mathscr{K}_{n, N}^{0,0}(-1,-1) \leqslant \sum_{k=0}^{n} \frac{2 k+1}{2} \exp \left\{\frac{4 k^{2}}{N-k}\right\} \leqslant \gamma(a) n^{2},
$$

где положительное $\gamma(a)$ зависит только от числа $a$. 
4. ДОКАЗАТЕЛЬСТВО ТЕОРЕМЫ. ПоЛожим

$$
\widehat{p}_{n}(x)=\frac{b_{n}(x-1)}{b_{n}(-1)}
$$

где $b_{n}(x)$ - многочлен, определенный равенством (12). Из свойств (11)-(13) следует, что многочлен (25) реализует нижнюю грань (1) среди всех многочленов $p_{n} \in \widehat{H}_{n}$, т.е.

$$
q(n, N)=\sum_{j=1}^{N} \widehat{p}_{n}^{2}(j)=\frac{1}{b_{n}^{2}(-1)} \sum_{j=0}^{N-1} b_{n}^{2}(j)=\frac{N}{2 \mathscr{K}_{n, N}^{0,0}(-1,-1)} .
$$

Здесь мы приняли во внимание тот факт, что при $\alpha=\beta=0$ весовая функция $\mu(x)=2 / N$ (см. (6)).

Если $n \leqslant a \sqrt{N}$, то из $(26)$ и $(24)$ имеем

$$
q(n, N) \geqslant \frac{N}{2 \gamma(a) n^{2}} \geqslant \frac{1}{2 \gamma a^{2}}=c(a)
$$

и, тем самым, оценка (2) доказана. Чтобы доказать соотношения (3)-(5) заметим, что величина $q(n, N)$ не возрастает с ростом $n$; в частности, $q(n, N)=0$ при $n \geqslant N$. Поэтому достаточно рассмотреть случай, когда $n \leqslant N / 4$ и $n / \sqrt{N} \rightarrow \infty$. Итак, пусть $n \leqslant N / 4$. Тогда для $0 \leqslant k \leqslant n$ имеем

$$
\frac{k^{2}}{2(N+k)}-\frac{k^{3}}{N^{2}}-\frac{k^{2}}{2 N(N-k)} \geqslant \frac{k^{2}}{2(N+N / 4)}-\frac{k^{2}}{4 N}-\frac{1}{24}=\frac{3 k^{2}}{20 N}-\frac{1}{24} .
$$

Поэтому из (22) следует, что

$$
\begin{aligned}
\mathscr{K}_{n, N}^{0,0}(-1,-1) & \geqslant \frac{1}{2} \sum_{k=1}^{n} k \exp \left\{\frac{3 k^{2}}{20 N}-\frac{1}{24}\right\}>\frac{1}{2 e^{1 / 24}}\left(\sum_{k=1}^{n-1} k+n \exp \left\{\frac{3 n^{2}}{20 N}\right\}\right) \\
& =\frac{1}{2 e^{1 / 24}}\left(\frac{(n-1) n}{2}+n \exp \left\{\frac{3 n^{2}}{20 N}\right\}\right) .
\end{aligned}
$$

Сопоставляя (26) и (27), приходим к оценке (4). Как уже отмечалось, $q(n, N)$ по переменной $n$ не возрастает, значит (5) вытекает из (4). Теорема доказана полностью.

ЗАмечАниЕ. Нижняя оценка (2) величины $q(n, N)$ может быть получена с помощью методов работ [2] и [3]. Другой способ получения оценки (2), путем сведения рассматриваемой задачи $\mathrm{K}$ некоторой двойственной, автору сообщил К. И. Осколков.

Автор признателен Б. С. Кашину и К. И. Осколкову за обсуждение результатов данной заметки и полезные замечания.

\section{СПИСОК ЦИТИРОВАННОЙ ЛИТЕРАТУРЫ}

1. Шарапудинов И. И. Многочлены, ортогональные на сетках. Теория и приложения. Махачкала: Изд-во ДГПУ, 1997. 2. Coppersmith D., Rivlin T. J. // SIAM J. Math. Anal. 1992. V. 23. Р. 970-983. 3. Шарапудинов И. И. // Матем. сб. 1996. Т. 187. №1. С. 143-160. 